\title{
Growth Hormone-Releasing Hormone Receptor Antagonist Modulates Lung Inflammation and Fibrosis due to Bleomycin
}

\author{
Chongxu Zhang ${ }^{1} \cdot$ Renzhi Cai $^{1} \cdot$ Aaron Lazerson $^{3} \cdot$ Gaetan Delcroix $^{1} \cdot$ Medhi Wangpaichitr $^{1} \cdot$ Mehdi Mirsaeidi $^{1,2}$. \\ Anthony J. Griswold ${ }^{4}$ Andrew V. Schally ${ }^{1,2,5} \cdot$ Robert M. Jackson $^{1,2,6}$ (1)
}

Received: 9 May 2019 / Accepted: 29 July 2019 / Published online: 7 August 2019

(c) The Author(s) 2019

\begin{abstract}
Purpose Growth hormone-releasing hormone (GHRH) is a 44-amino acid peptide that regulates growth hormone (GH) secretion. We hypothesized that a GHRH receptor (GHRH-R) antagonist, MIA-602, would inhibit bleomycin-induced lung inflammation and/or fibrosis in C57B1/6J mice.

Methods We tested whether MIA-602 (5 $\mu \mathrm{g}$ or vehicle given subcutaneously [SC] on days 1-21) would decrease lung inflammation (at day 14) and/or fibrosis (at day 28) in mice treated with intraperitoneal (IP) bleomycin (0.8 units on days $1,3,7,10,14$, and 21). Bleomycin resulted in inflammation and fibrosis around airways and vessels evident histologically at days 14 and 28 .

Results Inflammation (histopathologic scores assessed blindly) was visibly less evident in mice treated with MIA-602 for 14 days. After 28 days, lung hydroxyproline (HP) content increased significantly in mice treated with vehicle; in contrast, lung HP did not increase significantly compared to naïve controls in mice treated with GHRH-R antagonist. GHRH-R antagonist increased basal and maximal oxygen consumption of cultured lung fibroblasts. Multiple genes related to chemotaxis, IL-1, chemokines, regulation of inflammation, and extracellular signal-regulated kinases (ERK) were upregulated in lungs of mice treated with bleomycin and MIA-602. MIA-602 also prominently suppressed multiple genes related to the cellular immune response including those for T-cell differentiation, receptor signaling, activation, and cytokine production.

Conclusions MIA-602 reduced lung inflammation and fibrosis due to bleomycin. Multiple genes related to immune response and T-cell functions were downregulated, supporting the view that MIA-602 can modulate the cellular immune response to bleomycin lung injury.
\end{abstract}

Keywords Idiopathic pulmonary fibrosis · Growth hormone-releasing hormone $\cdot$ MIA-602 $\cdot$ Bleomycin

Robert M. Jackson

rjackson2@med.miami.edu

1 Research Service, Miami VAHS, Miami, FL 33125, USA

2 Department of Medicine, University of Miami, Miami, FL 33101, USA

3 Department of Comparative Pathology, University of Miami, Miami, FL 33101, USA

4 Dr. John T. MacDonald Foundation Department of Human Genetics, University of Miami, Miami, FL 33101, USA

5 Department of Pathology and Sylvester Cancer Center, University of Miami Miller School of Medicine, Miami, FL 33101, USA

6 Research Service, Miami VAHS, 1201 NW 16th Street, Miami, FL 33125, USA

\section{Introduction}

Idiopathic pulmonary fibrosis (IPF) is the paradigm of fibrosing interstitial lung diseases. It occurs more commonly in aging males, and often has a limited survival time of 3-5 years (median 3.8 years) after diagnosis [1]. Although the disease is of unknown etiology, it is clearly related to specific genetic abnormalities (e.g., MUC5B, SFTPC, and others) and environmental factors (e.g., dust and smoking) [2]. In response to injury, fibroblasts proliferate and migrate into the lung. They synthesize extracellular matrix, providing a platform for further cellular growth [3]. Myofibroblasts secrete cytokines, such as TGF- $\beta$, with autocrine and paracrine effects that drive fibrosis in the lung [4].

Growth hormone-releasing hormone (GHRH) is secreted primarily from the hypothalamus, but various other tissues 
can produce it locally [5]. GHRH stimulates the secretion and release of growth hormone $(\mathrm{GH})$ by the pituitary and in turn regulates the secretion of $\mathrm{GH}$ and insulin-like growth factor 1 (IGF-1) through the pituitary GH/hepatic IGF-1 axis [6]. We have found expression of pituitary-type GHRH receptor (pGHRH-R) in normal human and IPF lung tissue by western blotting, suggesting that GHRH or GH could participate in lung development, growth, and repair [7].

GHRH belongs to a peptide family that includes glucagon, secretin, vasoactive intestinal peptide (VIP), and pituitary adenylate cyclase-activating peptide (PACAP) [5]. GHRH-R antagonists exert growth-inhibitory effects in cancers in vitro and in vivo [8-10], in addition to having anti-inflammatory and anti-oxidative effects [11].

Human fibroblasts express GHRH receptors, which stimulate proliferation of fibroblasts through GH/IGF-1-mediated signaling. When skin wounds in mice are exposed to GHRH agonist, fibroblasts increase and repair of epithelium is accelerated [12]. GHRH stimulates the expression of $\alpha$-smooth muscle actin ( $\alpha$ SMA), which confers contractile activity in myofibroblasts [13]. In addition to its effects on GH and IGF-1, GHRH-R antagonist MIA-602 inhibits signaling pathways, including PAK1-STAT3/NF- $\mathrm{KB}$ in gastric cancer cells, suggesting it could modulate inflammatory and fibrotic processes [14].

We therefore used an established bleomycin model of lung inflammation and fibrosis in C57/B16 mice and synthetic GHRH receptor antagonist MIA-602 to test whether inhibition of GHRH receptors would limit inflammation and/ or fibrosis.

\section{Methods}

\section{Materials}

The GHRH-R antagonist, MIA-602 (MW 4843), was synthesized (PhAC-Ada, Tyr D-Arg, Asp, Ala, Ile, 5FPhe, Thr, Ala, Har, Tyr(Me), His, Orn, Val, Leu, Abu, Gin, Leu, Ser, Ala, His, Orn, Leu, Leu, Gin, Asp, Ile, Nle, D-Arg, Har $\mathrm{NH}_{2}$ ) at the Miami VAHS by solid phase methods and purified by HPLC.

\section{Experimental Animals}

We used 8-week-old C57Bl/6J male mice (The Jackson Laboratory, Bar Harbor, ME) in these experiments. Mice weighed about $26 \mathrm{~g}$ at the start. This protocol was approved by the Miami VAHS Animal Studies Subcommittee (IACUC) and it conformed to PHS Policy. Mice were randomly allocated to the experimental groups and housed in identical filter top cages in a ventilated rack. Mice were exposed to a 12-h light/dark cycle and had free access to standard laboratory chow and water.

\section{Bleomycin and GHRH-R Antagonist Treatment}

All mice were treated with 0.8 units of bleomycin (Bleomycin for Injection USP, Hospira, Lake Forest, IL) intraperitoneally (IP) on days 1, 3, 7, 10, 14, and 21 [15]. Mice (randomly assigned) were simultaneously treated subcutaneously (sc) with MIA-602 (5 $\mu \mathrm{g} /$ day $)$ or its vehicle $(100 \mu \mathrm{L} /$ day) [14]. MIA-602 was dissolved in DMSO (ACS grade; Sigma-Aldrich) and diluted 1:500 in normal saline for daily sc injection on days 0-21.

\section{Tissue Preparations}

Mice were killed by $\mathrm{CO}_{2}$ inhalation before any treatment, or on days 14 or 28 for micro-CT scans of lungs and harvesting of lung tissue [16]. The right mainstem bronchus was ligated and the right lung removed and frozen at $-80{ }^{\circ} \mathrm{C}$ for hydroxyproline (HP) assays. The left lung was filled with $10 \%$ buffered formalin at $\sim 25 \mathrm{~cm} \mathrm{H}_{2} \mathrm{O}$ pressure, the bronchus ligated, and the lung fixed in formalin. Fixed lungs were embedded in wax and $5 \mu \mathrm{m}$ sections stained with hematoxylin and eosin (H\&E) or Masson's trichrome stain.

\section{Lung Histopathology}

Fibrosis was quantified in trichrome-stained sections using a modification of the Ashcroft score that describes grades of fibrosis [17]. Ten randomly chosen fields on each slide were scored as described and averaged. Inflammation in lung tissue was quantified in H\&E-stained sections.

\section{Hydroxyproline Assay}

Right lungs were weighed and homogenized in 10 volumes of distilled water. Homogenates were hydrolyzed in $12 \mathrm{M}$ $\mathrm{HCl}$ at $120^{\circ} \mathrm{C}$ for $3 \mathrm{~h}$ as previously described [18]. Hydroxyproline was measured colorimetrically $(560 \mathrm{~nm})$ after hydrolysis using an assay kit (MAK008, Sigma-Aldrich, St. Louis, MO).

\section{Micro-CT}

Mice in each group were assessed by micro-CT scans (Bruker SkyScan 1176 Low-Dose Micro-CT, Knotich, Belgium) of the lungs after $\mathrm{CO}_{2}$ inhalation. A tracheostomy tube was inserted and lungs inflated with air. Scans were examined qualitatively to confirm development of infiltrates and reticular densities. 


\section{Lung Fibroblasts}

Newborn mouse lung fibroblasts (Mlg 2908) were obtained from the American Type Culture Collection, Manassas, VA and cultured in Eagle's minimal essential medium. Cells were grown in 6-well plates before incubation with 1 or $5 \mu$ M MIA- 602 or vehicle, RNA isolation, and oxygen consumption measurements.

\section{Annexin V-Propidium lodide Assay}

Apoptosis and necrosis were assessed with annexin V/propidium iodide staining (Annexin V: FITC Assay Kit, Bio Rad, Hercules, CA 94547) of lung fibroblasts incubated in 0,1 , or $5 \mu \mathrm{M}$ MIA-602 for $24 \mathrm{~h}$ [19]. Culture medium and trypsinized cells were collected and centrifuged at $400 \times g$ for $5 \mathrm{~min}$. The pellet was resuspended in $100 \mu \mathrm{L}$ annexin V/propidium iodide. The suspension was incubated at $37{ }^{\circ} \mathrm{C}$ for $20 \mathrm{~min}$, then washed with PBS, and resuspended in $500 \mu \mathrm{L}$ PBS and fluorescence quantified by Beckman Coulter Life Sciences CytoFLEX benchtop flow cytometer (Beckman Coulter, Inc., Brea, CA).

\section{RNA Isolation}

RNA was extracted from fixed lung tissue in paraffin blocks using a Quick-RNA ${ }^{\mathrm{TM}}$ FFPE Kit (R1008, Zymo Research, Irvine, CA) following the manufacturer's protocol [20]. Samples were deparaffinized, digested with proteinase $\mathrm{K}$, and decrosslinked at $65{ }^{\circ} \mathrm{C}$ for $15 \mathrm{~min}$. RNA lysis buffer was added and mixed with ethanol. The mixtures were transferred to spin columns to isolate total RNA.

RNA was extracted from cultured fibroblasts using a Direct-zol RNA MicroPrep kit (R2060; Zymo Research, Irvine, CA). Cells were washed with PBS and lysed in TRI reagent ${ }^{\mathrm{TM}}$, then purified using Direct-zol RNA columns. DNase I treatment was done in columns and RNA eluted in DNase/Rnase-free water.

\section{Cellular Respiration}

We measured effects of MIA-602 on mouse lung fibroblast oxygen consumption using the Agilent Seahorse XF Cell Mito Stress Test (Agilent Technologies, Santa Clara, CA) [21]. Fibroblasts were incubated with vehicle, 1 or $5 \mu \mathrm{M}$ MIA-602 for $24 \mathrm{~h}$ before measurement of oxygen consumption. One day before assay 80,000 fibroblasts were seeded into Seahorse 24-well plates ( $n=6$ wells per condition). Basal respiration was established and oligomycin, FCCP, and rotenone plus antimycin A were added sequentially to measure ATP production, uncoupled respiration, and non-mitochondrial oxygen consumption [22].

\section{RNA-seq and Pathway Analyses}

At least $10 \mathrm{ng}$ of total RNA was used as input for the KAPA RNA HyperPrep Kit with RiboErase (HMR) to create ribosomal RNA-depleted sequencing libraries, including sample indexing, to allow for multiplexing. Cluster generation and sequencing was done on the Illumina cBOT and HiSeq 3000 using reagents provided by Illumina, finally generating $>32$ million single-end 100 base reads per sample.

We created de-multiplexed FASTQ files with Illumina supplied scripts in BCL2FASTQ software (v2.17). Illumina adapters were trimmed using the Trim Galore! package and aligned to the mouse reference genome (mm10) with STAR aligner (v2.5.0a) with default alignment parameters [23].

Gene counts were normalized using trimmed mean of M-values (TMM) method. Differential expression between groups was calculated with the exact test implemented in edgeR [24].

Pathway enrichment analyses were completed using Enrichr online and DAVID bioinformatics resource [25, 26].

\section{Data Analysis}

Data are reported as arithmetic means \pm SEM or SD as indicated. Medians and 25-75th percentiles were used to describe the distribution of histopathological scores. ANOVA followed by Dunnett's test or the Bonferroni correction was used for multiple comparisons with a control or among groups [27]. We planned at least six mice available at each time point in each group, so that we would have sufficient power to detect a $20 \%$ change in lung hydroxyproline given an assumed coefficient of variance of $0.2 . p \leq 0.05$ was considered significant.

\section{Results}

\section{Micro-CT Scans}

Mice treated with vehicle developed patchy infiltrative densities that persisted to day 28. Mice that received bleomycin plus MIA-602, the GHRH-R antagonist, appeared to have less prominent infiltrative densities in their lungs.

\section{Lung Hydroxyproline Contents}

Lung HP content did not increase significantly after 14 days of intermittent treatment with bleomycin in mice receiving MIA-602 or vehicle. However, after 28 days, lung HP content increased significantly in bleomycin-treated mice that 


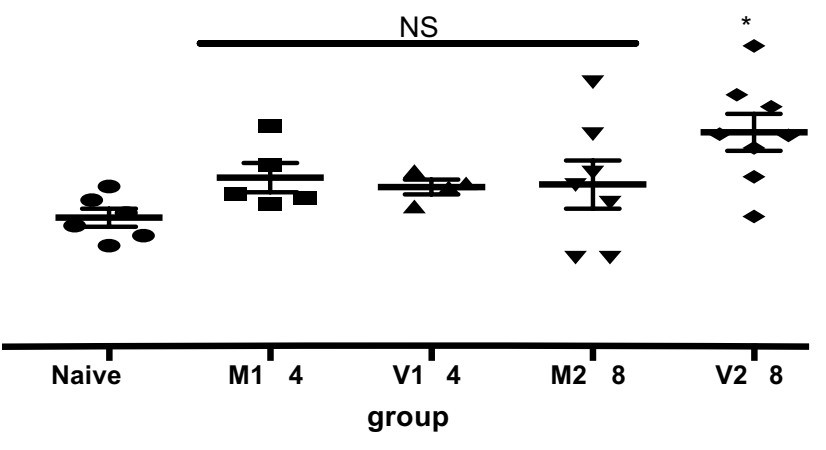

Fig. 1 Lung hydroxyproline content. Evaluation of lung hydroxyproline (HP) content to estimate changes in collagen due to bleomycin and the effect of MIA-602. Data shown are mean hydroxyproline contents of right lungs \pm SEM at 14 - and 28 -day time points. Normal C57Bl/6J mice $(n=6)$ had about $20 \mu \mathrm{g} \mathrm{HP}$ in the right lung. HP content increased significantly ( ${ }^{*} p=0.0060$ compared to Naive) after 28 days in mice treated with bleomycin and vehicle. No significant increase in HP content occurred in lungs of mice treated with bleomycin and the GHRH-R antagonist MIA-602. M14, MIA-602 group at 14 days $(n=5)$; V14, vehicle group at 14 days $(n=4)$; M28, MIA602 group at 28 days $(n=7) ; \mathrm{V} 28$, vehicle group at 28 days $(n=8)$

received vehicle, but not in bleomycin-treated mice that received MIA-602 on days $1-21$. The data are summarized in Fig. 1.

\section{Lung Histopathology}

After 14 days of intermittent bleomycin, inflammation and patchy mild fibrosis were qualitatively evident in mouse lungs. More extensive fibrosis and fewer inflammatory cells were evident after 28 days. Both inflammatory changes and fibrosis appeared decreased in mice that received MIA602 in addition to bleomycin. Representative examples are shown in Fig. 2.

MIA-602 appeared to reduce inflammation after 14 days in lungs of bleomycin-treated mice (0.9 [median]; 0.6-1.2 [25-75th percentile]; $n=4)$, compared to mice receiving vehicle $(1.4 ; 0.9-1.8 ; n=4)$. MIA-602 appeared to reduce fibrosis after 28 days in lungs of bleomycin-treated mice that received MIA-602 $(2.0 ; 1.4-2.6 ; n=5)$, but not in mice treated with vehicle $(2.2 ; 2.1-2.9 ; n=8)$.

\section{Lung Fibroblast Response to MIA-602}

Lung fibroblasts were exposed to MIA-602 or vehicle in vitro for $24 \mathrm{~h}$ before annexin V/PI assay. MIA-602 caused predominantly cytolytic cell death $(1 \mu \mathrm{M}, 9.3 \pm 1.1 \% ; 5 \mu \mathrm{M}$, $34.9 \pm 2.5 \% ; p=0.0002)$ rather than apoptosis.

\section{Cellular Respiration}

In vitro, MIA-602 at $5 \mu \mathrm{M}$ concentration in complete medium increased fibroblast basal respiration and maximal respiration (after FCCP), compared to vehicle. Both 1 and $5 \mu \mathrm{M}$ MIA-602 also appeared to increase non-mitochondrial respiration (after antimycin A and rotenone). These data are summarized in Fig. 3.

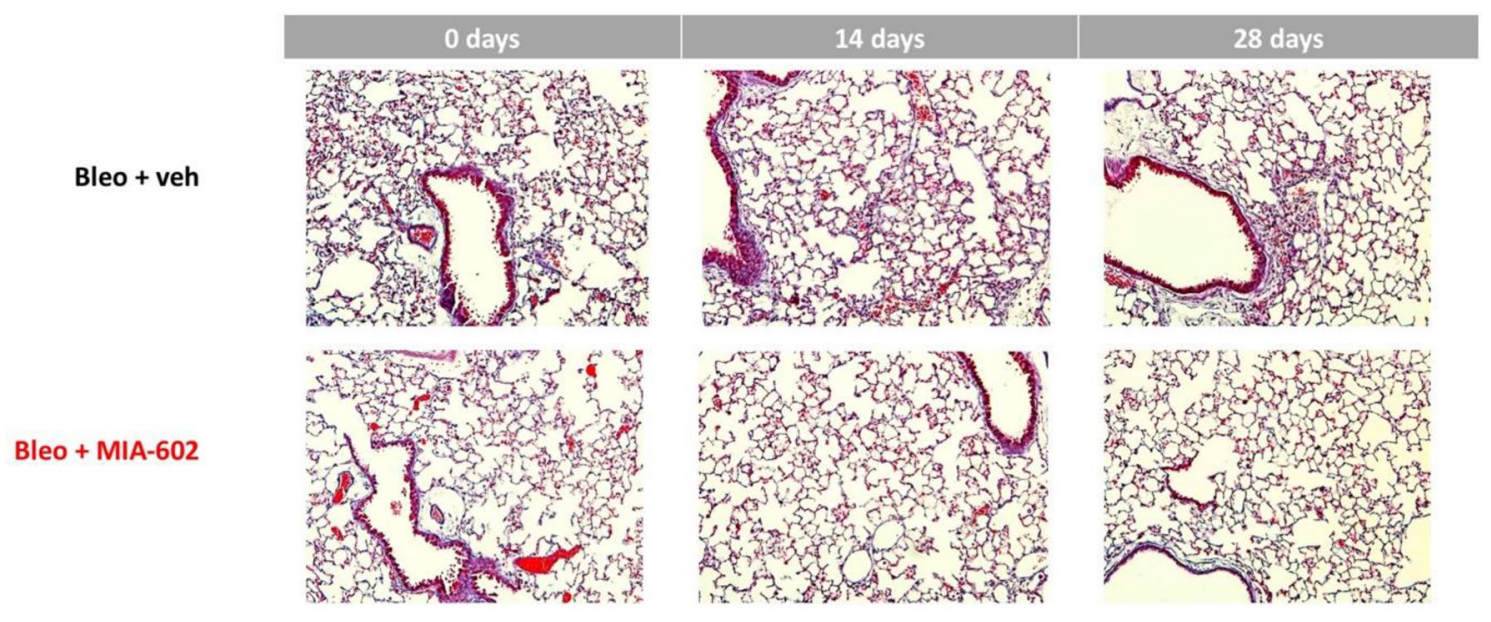

Fig. 2 Lung histopathology. Mouse lungs were inflated with buffered formalin to $25 \mathrm{~cm} \mathrm{H}_{2} \mathrm{O}$ pressure and fixed. $5 \mu \mathrm{m}$ sections were stained with Masson's trichrome stain and assessed semi-quantitatively for inflammation and fibrosis as described in the text. 14 days after bleomycin was started, cellular inflammation and early fibrosis was detected in lungs of mice treated with bleomycin and vehicle. Less inflammation appeared to be present in lungs of mice that received bleomycin and MIA-602 (middle panels). 28 days after bleomycin was started, increased fibrosis was evident in lungs of mice treated with bleomycin and vehicle. Less fibrosis appeared to be present in lungs of mice that received bleomycin and MIA-602, the growth hormone receptor antagonist 
Fig. 3 GHRH-R antagonist increases lung fibroblast basal and maximal oxygen consumption. Panel A shows a representative mitochondrial stress assay of mouse lung fibroblasts (data shown are means \pm SD of 6 wells in each condition) exposed to vehicle (light gray), $1 \mu \mathrm{M}$ (medium gray) or $5 \mu \mathrm{M}$ (black) MIA-602 for $24 \mathrm{~h}$ before measurements of oxygen consumption with oligomycin, FCCP, antimycin A, and rotenone. $5 \mu \mathrm{M}$ MIA-602 increased basal oxygen consumption (* $p=0.0403$ compared to vehicle) and maximal uncoupled respiration $(* * p<0.0001)$ of normal mouse lung fibroblasts. Panel B shows that $5 \mu \mathrm{M}$ MIA-602 increased both basal respiration $\left({ }^{*} p=0.0125\right)$ and spare respiratory capacity $(* * p<0.0001)$
A

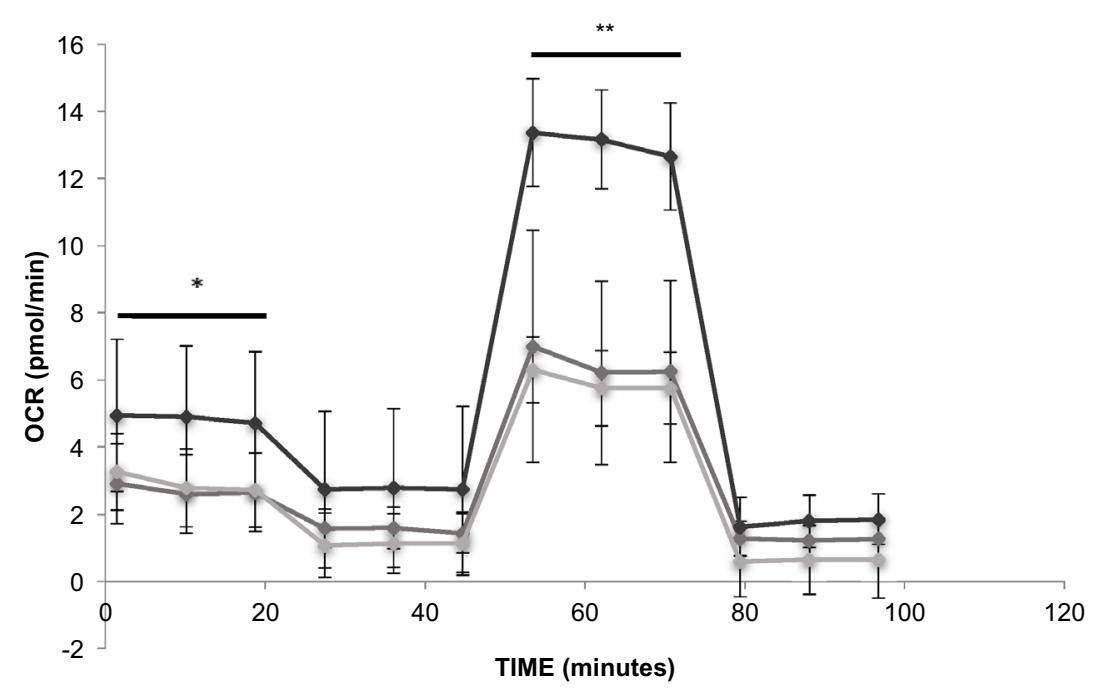

B

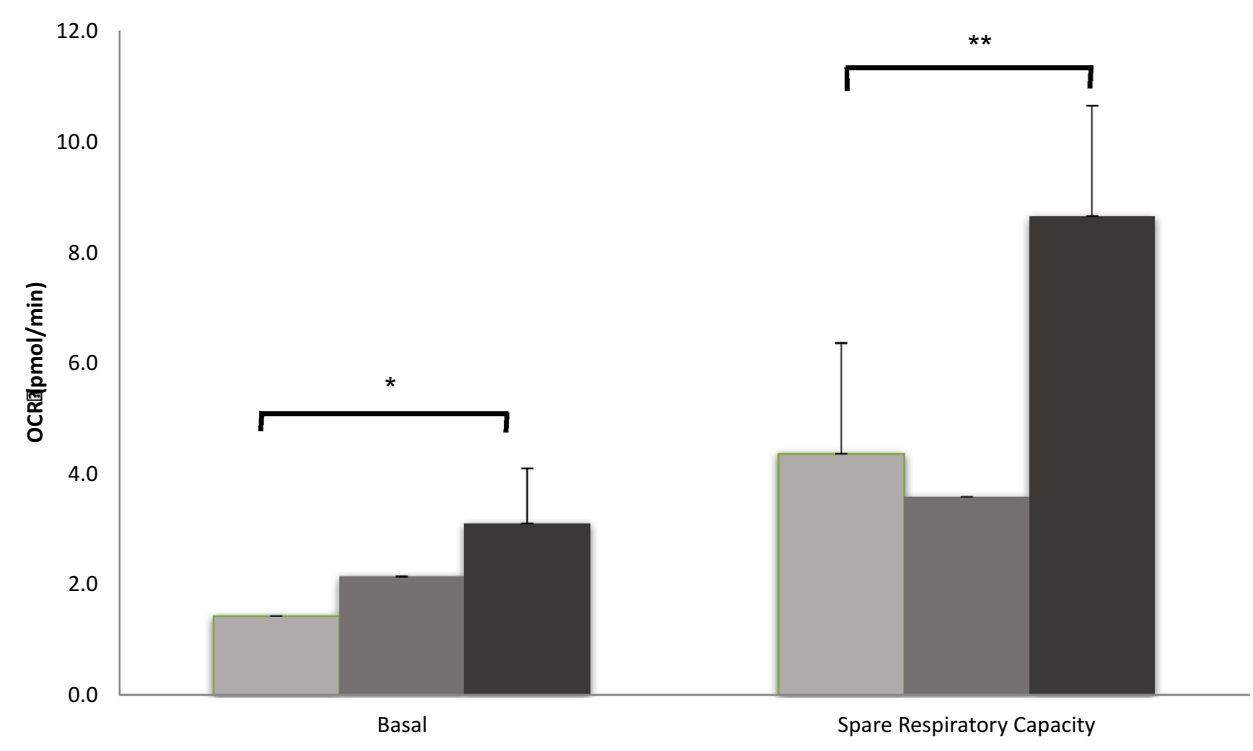

\section{RNA-seq Gene Expression}

We explored the effects of MIA-602 or vehicle on gene expression in lungs from mice treated in vivo with bleomycin for 28 days (data not shown). Several physiologically relevant genes were expressed differently after treatment with bleomycin (absolute fold change $>1.5$ and FDR $<0.01$ ). Specifically, after 28 days during which bleomycin was administered, genes related to the extracellular matrix, Wnt regulation and signaling, and the extracellular region were upregulated, consistent with known effects of bleomycin. We found several relevant genes to be downregulated by bleomycin, including those related to lung morphogenesis and development, extracellular matrix organization, and alveolar septal development.
Transcriptome profiles then showed numerous genes expressed differently after treatment with MIA-602. Inversely modulated genes were highly enriched in pathways related to the adaptive immune response, T-cell differentiation, T-cell signaling, extracellular matrix organization, T-cell activation and differentiation, and cytokine production, all consistent with the putative anti-inflammatory and anti-fibrotic effects of MIA-602. The ten most differentially expressed genes detected in lung tissue treated with MIA602 compared to vehicle treatment are shown in Table 1. Those genes differentially expressed in fibrotic lungs from bleomycin-exposed mice treated with MIA-602 compared to vehicle are displayed as a heat map in Fig. 4.

Similarly, we directly tested the in vitro effects of 1 and $5 \mu \mathrm{M}$ MIA-602 on normal mouse lung fibroblasts (not 
A

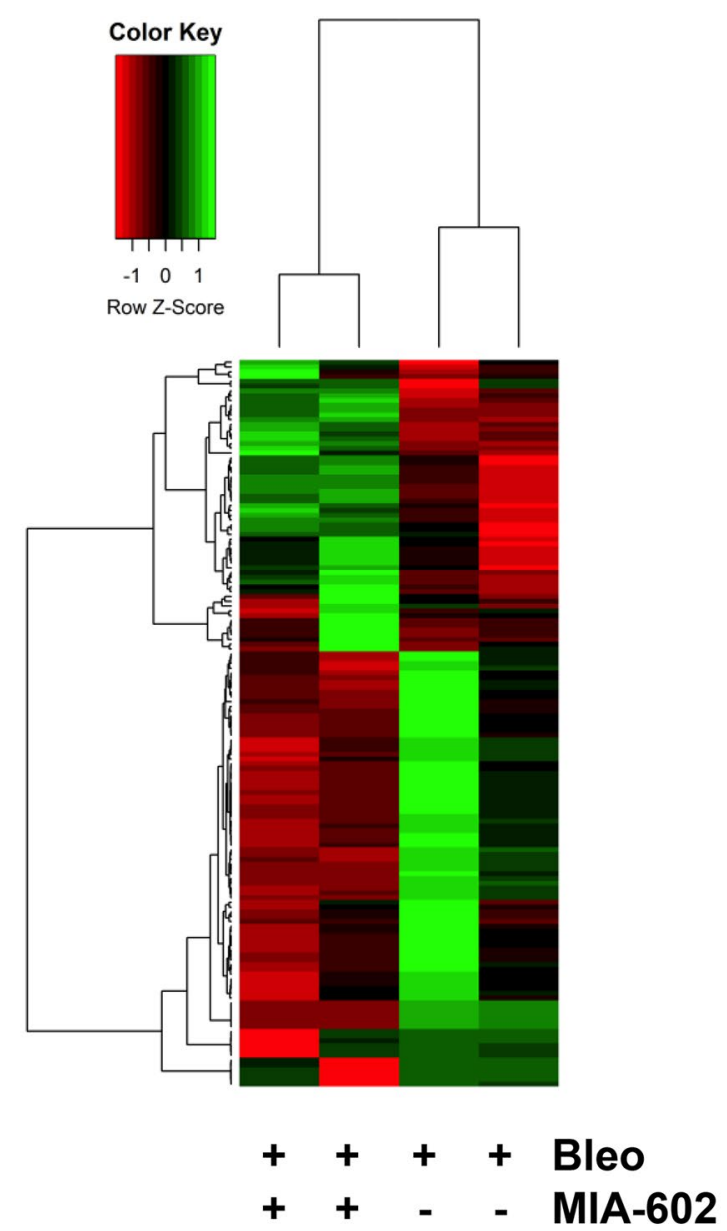

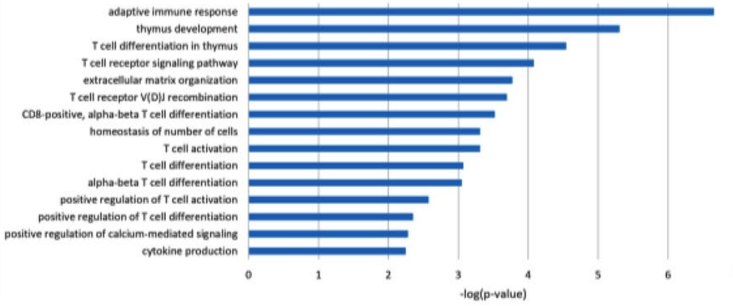

Fig. 4 Transcriptomic analysis of mouse lung tissue RNA after treatment of mice in vivo with bleomycin and MIA-602 or vehicle. Panel a Heat map analysis showing differential gene expression in lungs from mice treated with bleomycin for 28 days that also received MIA$602(+)$ or vehicle $(-)$ for the first 21 days. The left two columns show gene expression in bleomycin- and MIA-602-treated mice,

exposed to bleomycin) and found significant downregulation of genes involved in collagen fibril organization, cell-matrix adhesion, and elastic fiber assembly, all consistent with the demonstrated anti-fibrotic effects of MIA-602. Upregulated fibroblast genes included those related to protein kinase activity, the JAK-STAT cascade, cell cycle, and DNA replication including histones.

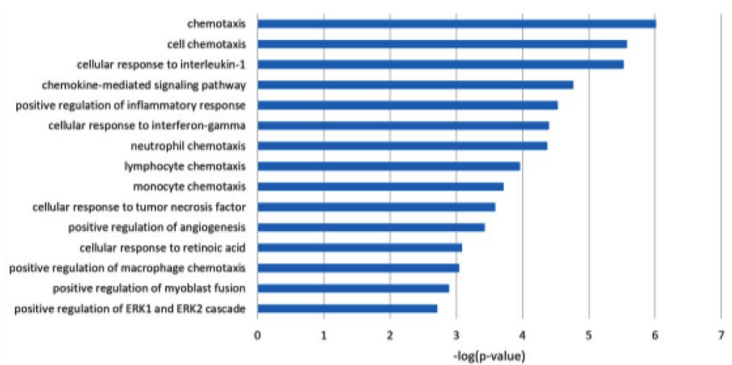

whereas the right two columns show gene expression in bleomycinand vehicle-treated lungs. Panel b Pathway analysis showing differentially expressed genes (downregulated, left panel; upregulated, right panel) in lungs from bleomycin-treated mice also treated with MIA-602 compared to those also treated with vehicle

\section{Discussion}

Pathophysiological GH secretion and IGF-1 activation have growth-promoting effects in the lung resulting in increased alveolar size [28]. IGF-1 itself increases $\alpha$-smooth muscle actin in lung fibroblasts and promotes a myofibroblast phenotype. The pituitary-type GHRH receptor is present in both 
Table 1 Clusters of the differentially expressed genes and their ontology between bleomycin-induced pulmonary fibrosis treated vs. untreated with MIA-602

\begin{tabular}{|c|c|c|c|}
\hline Name & Gene list & $p$ value & Adjusted $p$ value \\
\hline T-cell differentiation (GO:0030217) & CD4, CD8A, RAG2, RAG1 & 0.000003374 & 0.0007392 \\
\hline T-cell activation (GO:0042110) & CD4, CD8A, CD3G, CD3E, LAT & 0.000003777 & 0.0007392 \\
\hline T-cell receptor signaling pathway (GO:0050852) & PSMB11, CD4, THEMIS, CD3G, CD3E, LAT & 0.000004630 & 0.0007392 \\
\hline Antigen receptor-mediated signaling pathway (GO:0050851) & PSMB11, CD4, THEMIS, CD3E, LAT & 0.00006007 & 0.007193 \\
\hline V(D) J recombination (GO:0033151) & RAG2, RAG1 & 0.0001910 & 0.01829 \\
\hline $\begin{array}{l}\text { Enzyme-linked receptor protein signaling pathway } \\
\text { (GO:0007167) }\end{array}$ & CD4, CD8A, NPPA, CD3A & 0.0002969 & 0.02370 \\
\hline T-cell differentiation in thymus (GO:0033077) & RAG2, RAG1 & 0.0004471 & 0.02677 \\
\hline Regulation of leukocyte cell-cell adhesion (GO:1903037) & CD4, LAT & 0.0004471 & 0.02677 \\
\hline Regulation of lymphocyte activation (GO:0051249) & CD4. LAT & 0.0006144 & 0.03270 \\
\hline Lymphocyte differentiation (GO:0030098) & CD4, RAG2, RAG1 & 0.0009974 & 0.04777 \\
\hline
\end{tabular}

normal and IPF lung tissue [7], suggesting that local secretion of GH may occur physiologically and have direct effects on lung tissue.

MIA-602 partially inhibits both lung inflammation and fibrosis, assessed histopathologically and biochemically, after intraperitoneal bleomycin. RNA-seq data, importantly, show suppression of the adaptive immune response, T-cell differentiation and activation, and cytokine production by MIA-602 in bleomycin-treated mouse lungs. The effects of the GHRH-R antagonist we observe have implications for fibrosing lung diseases in humans, and they could, importantly, reveal novel pathways amenable to clinical drug development [29].

We initially confirmed development of lung infiltrates due to bleomycin with micro-CT scans, inflammation, and fibrosis with histopathological examination, and increased collagen with biochemical assays. Similar models have been used successfully in the development of anti-fibrotic drugs, including pirfenidone and nintedanib.

Senescent fibroblasts that display respiratory abnormalities, indicating mitochondrial damage, express both STAT3 and p21 as markers of the senescent phenotype [30]. MIA-602 downregulates p21-activated kinase and STAT3 and NFאB in gastric cancer cells [14]. GHRH antagonists like MIA-602 could modulate the senescent phenotype leading to fibrosis, and conceivably be one of the mechanisms that lessens the fibrotic response in this model.

In vitro, MIA-602 at micromolar concentrations increased basal and maximal mitochondrial respiration, and it resulted in marked cytolytic death of mouse lung fibroblasts. Mitochondrial dysfunction and loss of apoptotic potential occur in fibroblasts from IPF lungs, and enhancement of mitochondrial function itself by MIA-602 at lower concentrations in vivo might modulate fibrosis by maintaining the capacity for mitophagy and apoptosis [31].
GHRH-R antagonists decrease lipid peroxidation, protein carbonyls, and nitrotyrosine in prostate cancer cells [32], indicating antioxidant effects that would augment their other anti-inflammatory effects [33]. Both GH and IGF-1 stimulate neutrophil superoxide $\left(\mathrm{O}_{2}{ }^{-}\right)$production [9]. Since GHRH-R antagonists inhibit GH secretion and IGF-1 activation [34], it could be predicted that they would inhibit both $\mathrm{O}_{2}{ }^{-}$and hydrogen peroxide $\left(\mathrm{H}_{2} \mathrm{O}_{2}\right)$ release during the inflammatory phase of injury [35]. Cellular levels of oxidant stress are decreased by MIA-602, and its antioxidant effect would limit redox signaling in response to receptor ligation [11].

MIA-602 disrupts the PI3K/AKT pathway in several experimental systems. PI3K/AKT signaling is involved in the pathogenesis of bleomycin-induced fibrosis [36], and suppression of the PI3K/AKT pathway by GHRH antagonist could also lessen lung fibrosis. GHRH clearly appears involved in the lung's response to treatment with bleomycin and subsequent healing.

Genes related to the extracellular matrix and the Wnt pathway were over expressed in fibrotic lungs from mice treated with bleomycin, compared to gene expression in naïve controls [37]. In contrast, genes related to epithelial tube branching, lung morphogenesis, and lung development were under expressed after bleomycin and development of fibrosis. Genes related the immune response, cellular adhesion, and remodeling, and T-cell signaling were also found to be upregulated in lungs from rats treated with TGF-ß [38].

$\mathrm{T}$ cells play a central role in the lungs of patients with pulmonary fibrosis [39]. T-cell receptors and costimulatory molecules are required for activation of $\mathrm{T}$ cells and in development of inflammation-driven lung fibrosis [40]. We found that downregulation of T-cell receptor complex genes (CD3E, CD3G, CD4, and CD8A) had the highest associations in pathway analyses. MIA-602 may thus play an important role in lung tissue by modification of 
T-cell signaling and potentially reducing inflammation and fibrosis.

These data show that GHRH-R is present in human lungs; and, in a relevant in vivo model, lung fibrosis is modulated by its inhibition. Functional findings implicate GHRH and GH in fibrosing lung disease, and they are consistent with demonstrated effects of the GHRH-R antagonist on mitochondrial respiration and fibroblast cytotoxicity. MIA-602 inhibits intracellular signaling pathways, including p21-activated kinase/STAT3/NFKB and PI3K/AKT, in addition to having intrinsic antioxidant activity. Further, it could support mitochondrial function and maintain autophagy, minimizing fibrosis. These findings suggest the merit of further investigations of GHRH-R antagonists like MIA-602 regarding their mechanisms of action and applications in management of pulmonary fibrosis.

Acknowledgements This work was supported by the Miami VAHS Research Service (RJ), a Distinguished Medical Scientist Award (AVS), and Merit Review grant (MW) from the VA Office of Research and Development. The authors thank Fei Tang, $\mathrm{PhD}$, for biostatistical assistance in experimental design and analysis of the data, and they thank Rocio Acevedo for help in preparing the manuscript.

Author Contributions $\mathrm{CZ}$ conducted mouse and in vitro experiments, analyzed data, and edited manuscript. RC synthesized MIA-602 and related peptides, determined dosing, and reviewed manuscript. AL analyzed lung histopathology slides qualitatively and quantitatively. GD assisted with the acquisition of micro-CT images and their qualitative analysis. MW conducted Seahorse experiments, analyzed data, and assisted with writing the manuscript. MM assisted with in vivo experiments, analyzed RNA-seq data, completed pathway analyses, and assisted in writing the manuscript. AG did RNA-seq of mouse lung RNA, advised on data analysis, and edited the manuscript. AS suggested testing and provided MIA-602, assisted in experimental design, and thoroughly edited the manuscript. RJ planned, supervised, and assisted in experiments; reviewed experimental and statistical data; and wrote the manuscript.

Funding This study was funded by VA Distinguished Scientist Award, VA (Grant No. 1 I01 BX004371), and VA Research Service.

\section{Compliance with Ethical Standards}

Conflict of interest A.V.S. and R.C. are listed as co-inventors on patents on GHRH analogs, which were assigned to the University of Miami and Veterans Affairs Department.

Open Access This article is distributed under the terms of the Creative Commons Attribution 4.0 International License (http://creativeco mmons.org/licenses/by/4.0/), which permits unrestricted use, distribution, and reproduction in any medium, provided you give appropriate credit to the original author(s) and the source, provide a link to the Creative Commons license, and indicate if changes were made.

\section{References}

1. Lederer D, Martinez F (2018) Idiopathic pulmonary fibrosis. N Engl J Med 378:1811-1823

2. Schwartz D (2016) Idiopathic pulmonary fibrosis is a complex genetic disorder. Trans Am Clin Climatol Assoc 127:34-45

3. Herrera J, Henke C, Bitterman P (2018) Extracellular matrix as a driver of progressive fibrosis. J Clin Invest 128:45-53

4. Wei Y, Kim T, Peng D, Duan D, Gibbons D, Yamauchi M, Jackson J, Le Saux C, Calhoun C, Peters J, Derynck R, Backes B, Chapman H (2017) Fibroblast-specific inhibition of TGF$\beta 1$ signaling attenuates lung and tumor fibrosis. J Clin Invest 127:3675-3688

5. Kiaris H, Chatzistamou I, Papavassiliou A, Schally A (2011) Growth hormone-releasing hormone: Not only a neurohormone. Trends Endocrinol Metab 22:311-317

6. Hung C, Rohani M, Lee S, Chen P, Schnapp L (2013) Role of IGF-1 pathway in lung fibroblast activation. Respir Res 14:102

7. Jackson R, Ai L, Zhang C, Zhang X, Delcroix G, Lazerson A, Mirsaeidi M, Schally A (2018) Growth hormone-releasing hormone receptor antagonist modulates mouse lung inflammation and fibrosis due to bleomycin. Eur Respir J 52(suppl 62):OA5349

8. Perez R, Schally A, Vidaurre I, Ricon R, Block N, Rick F (2012) Antagonists of growth hormone-releasing hormone suppress in vivo tumor growth and gene expression in triple negative breast cancers. Oncotarget 3:988-997

9. Schally A, Varga J, Engel J (2007) Antagonists of growth hormone releasing hormone: and emerging new therapy for cancer. Nat Clin Pract Endocrinol Metab 4:33-43

10. Zarandi M, Cai R, Kovacs M, Popovics P, Szalontay L, Cui T, Sha W, Jaszberenyi M, Varga J, Zhang X, Block N, Rick F, Halmos G, Schally A (2017) Synthesis and structure-activity studies on novel analogs of human growth hormone releasing hormone (GHRH) with enhanced inhibitory activities on tumor growth. Peptides 89:60-70

11. Barbutis N, Schally A (2008) Antioxidant activity of GHRH antagonists in LNCaP human prostate cancer line. PNAS 105:20470-20475

12. Cui T, Jimenez J, Block N, Badiavas E, Rodriguez-Menocal L, Vila Granda A, Cai R, Sha W, Zarandi M, Perez R, Schally A (2016) Agonistic analogs of growth hormone-releasing hormone (GHRH) promote wound healing by stimulating the proliferation and survival of human dermal fibroblasts through ERK and AKT pathways. Oncotarget 7:52661-52672

13. Zhang X, Xing R, Chen L, Liu C, Miao Z (2016) PI3K/Akt signaling is involved in the pathogenesis of bleomycin-induced pulmonary fibrosis via regulation of epithelial-mesenchymal transition. Mol Med Rep 14(6):5699-5706

14. Gan J, Ke X, Jiang J, Dong H, Yao Z, Lin Y, Lin W, Wu X, Yan S, Zhuang Y, Chu W, Cai R, Zhang X, Cheung H, Block N, Pang C, Schally A, Zhang H (2016) Growth hormone-releasing hormone receptor antagonists inhibit human gastric cancer through downregulation of PAK1-STAT3/NF- $\mathrm{KB}$ signaling. Proc Natl Acad Sci USA 113:14745-14750

15. Collins S, Chan-Li Y, Oh M, Vigeland C, Limjunyawong N, Mitzner W, Powell JD, Horton MR (2016) Vaccinia vaccinebased immunotherapy arrests and reverses established pulmonary fibrosis. JCI Insight 1(4):1-13

16. Vande Velde G, Poelmans J, De Langhe E, Hillen A, Vanoirbeek J, Himmelreich U, Lories R (2016) Longitudinal micro-CT 
provides biomarkers of lung disease that can be used to assess the effect of therapy in preclinical mouse models and reveal compensatory changes in lung volume. Dis Model Mech 9:91

17. Hübner R, Gitter W, El Mokhtari N, Mathiak M, Both M, Bolte H, Freitag- Wolf S, Bewig B (2008) Standardized quantification of pulmonary fibrosis in histological samples. Biotechniques 44:507-517

18. Kennedy J, Chandler D, Jackson R, Fulmer J (1986) Reduction in bleomycin- induced lung hydroxyproline content by an iron chelating agent. Chest 89(3 Suppl):123S-125S

19. Rieger A, Nelson K, Konowalchuk J, Barreda D (2011) Modified annexin V/Propidium iodide assay for accurate assessment of cell death. J Vis Exp 50:e2597

20. Patel P, Selvarajah S, Guerard K, Bartlett J, Lapointe J, Berman D, Okello J, Park P (2017) Reliability and performance of commercial RNA and DNA extraction kits for FPPE tissue cores. PLoS ONE 12:e0179732

21. Divakaruni A, Paradyse A, Ferrick D, Murphy A, Jastroch M (2014) Analysis and interpretation of microplate-based oxygen consumption and pH data. Methods Enzymol 547:309-354

22. Wangpaichitr WuC, Li Y, Nguyen D, Kandemir H, Shah S, Chen S, Feun L, Prince J, Kuo M, Savaraj N (2017) Exploiting ROS and metabolic differences to kill cisplatin resistant lung cancer. Oncotarget 8(30):49275-49292

23. Dobin A, Davis C, Shlesinger F, Drenkow J, Zaleski C, Jha S, Batut P, Chaisson M, Gingeras T (2013) STAR: ultrafast universal RNA-seq aligner. Bioinformatics 29:15-21

24. Robinson M, McCarthy D, Smyth G (2010) edgeR: a Bioconductor package for differential expression analysis of digital gene expression data. Bioinformatics 26:139-140

25. Chen E, Tan C, Kou Y, Duan Q, Wang Z, Meirelles G, Clark N, Ma'ayan A (2013) Enrichr: interactive and collaborative HTML5 gene list enrichment analysis tool. BMC Bioinform 14:128

26. Huang W, Sherman B, Lempicki R (2009) Systematic and integrative analysis of large gene lists using DAVID bioinformatics resources. Nat Protoc 4:44-57

27. Kusuoka H, Hoffman $\mathbf{J}$ (2002) Advice on statistical analysis for circulation research. Circ Res 91:662-671

28. Garcia-Rio F, Pino J, Diez J, Ruiz A, Villasante C, Villamor J (2001) Reduction of lung distensibilty in acromegaly after suppression of growth hormone hypersecretion. Am J Respir Crit Care Med 164:852-857

29. Jenkins R, Moore B, Chambers R, Eickelberg O, Königshoff M, Kolb M, Laurent GJ, Nanthakumar CB, Olman M, Pardo A, Selman M, Sheppard D, Sime P, Tager A, Tatler A, Thannickal V, White E (2017) Use of animal models for the preclinical assessment of potential therapies for pulmonary fibrosis. Am J Respir Cell Mol Biol 56:667-679

30. Waters D, Blokland K, Pathinayake P, Wei Schuliga M, Jaffar J, Hansbro P, Prele C, Mutsaeres S, Bartlett N, Grainge C, Knight D (2019) STAT3 regulates the onset of oxidant-induced senescence in lung fibroblasts. Am J Resp Cell Molec Biol. https://doi. org/10.1165/rcmb.2018-03280C
31. Ryter S, Rosas I, Owen C, Martinez F, Choi M, Lee C, Elias J, Choi A (2018) Mitochondrial dysfunction as a pathogenic mediator of chronic obstructive pulmonary disease and idiopathic pulmonary fibrosis. Ann Am Thorac Soc 15(Suppl 4):S266-S272

32. Rekasi Z, Varga J, Schally A, Halmos G, Armatis P, Groot K, Czompoly T (2001) Antagonists of growth hormone-releasing hormone and vasoactive intestinal peptide inhibit tumor proliferation by different mechanisms: Evidence from in vitro studies on human prostatic and pancreatic cancers. Endocrinology 141:2120-2128

33. Ren J, Yu Q, Ma D, Liang W, Leung P, Ng T, Chu W, Schally A, Pang C, Chan S (2019) Growth hormone-releasing hormone receptor mediates cytokine production in ciliary and iris epithelial cells during LPS-induced ocular inflammation. Exp Eye Res 181:277-284

34. Fu Y, Arkins S, Wang B, Kelley K (1991) A novel role of growth hormone and insulin-like growth factor-1. J Immunol 146:1602-1608

35. Warwick-Davies J, Lowrie D, Cole P (1995) Growth hormone is a human macrophage activating factor. Priming of human monocytes for enhanced release of $\mathrm{H}_{2} \mathrm{O}_{2}$. J Immunol 154:1909-1918

36. Kral J, Kuttke M, Schrottmaier W, Birnecker B, Warszawska J, Wernig C, Paar H, Salzmann M, Sahin E, Brunner JS, Österreicher C, Knapp S, Assinger A, Schabbauer G (2016) Sustained PI3K activation exacerbates BLM- induced lung fibrosis via activation of pro-inflammatory and pro-fibrotic pathways. Sci Rep 6:23034. https://doi.org/10.1038/srep23034

37. Cabrera S, Selman M, Lonzano-Bolanos A, Konishi K, Richards T, Kaminski N, Pardo A (2013) Gene expression profiles reveal molecular mechanisms involved in the progression and resolution of bleomycin-induced lung fibrosis. Am J Physiol Lung Cell Mol Physiol 304:L593-L601

38. Huang X, Li L, Ammor R, Zhang Y, Wang Y, Ravi K, Thompson J, Jarai G (2019) Molecular characterization of a precision-cut lung rat lung slice model for the evaluation of antifibrotic drugs. Am J Physiol Lung Cell Mol Physiol 316:L348-L357

39. Simonian P, Roark C, Diaz del Valle F, Palmer B, Douglas IS, Ikuta K, Born W, O'Brien R, Fontenot A (2006) Regulatory role of gamma delta $\mathrm{T}$ cells in the recruitment of CD4+ and CD8+ $\mathrm{T}$ cells to lung and subsequent pulmonary fibrosis. J Immunol 177:4436

40. Elhai M, Avouac J, Hoffmann-Vold A, Ruzehaji N, Amiar O, Ruiz B, Brahiti H, Ponsoye M, Fréchet M, Burgevin A, Pezet S, Sadoine J, Guilbert T, Nicco C, Akiba H, Heissmeyer V, Subramaniam A, Resnick R, Molberg Ø, Kahan A, Chiocchia G, Allanore Y (2016) OX40L blockade protects against inflammation-driven fibrosis. Proc Natl Acad Sci USA 113:E3901-E3910

Publisher's Note Springer Nature remains neutral with regard to jurisdictional claims in published maps and institutional affiliations. 